\title{
Computational Identification of Essential Enzymes as Potential Drug Targets in Shigella flexneri Pathogenesis Using Metabolic Pathway Analysis and Epitope Mapping
}

\author{
Priyanka Narad, Himanshu, and Hina Bansal* \\ Amity Institute of Biotechnology, Amity University Uttar Pradesh, Sector 125, Noida-201303, U.P., India
}

\begin{abstract}
Shigella flexneri is a facultative intracellular pathogen that causes bacillary dysentery in humans. Infection with S. flexneri can result in more than a million deaths yearly and most of the victims are children in developing countries. Therefore, identifying novel and unique drug targets against this pathogen is instrumental to overcome the problem of drug resistance to the antibiotics given to patients as the current therapy. In this study, a comparative analysis of the metabolic pathways of the host and pathogen was performed to identify this pathogen's essential enzymes for the survival and propose potential drug targets. First, we extracted the metabolic pathways of the host, Homo sapiens, and pathogen, $S$. flexneri, from the KEGG database. Next, we manually compared the pathways to categorize those that were exclusive to the pathogen. Further, all enzymes for the 26 unique pathways were extracted and submitted to the Geptop tool to identify essential enzymes for further screening in determining the feasibility of the therapeutic targets that were predicted and analyzed using PPI network analysis, subcellular localization, druggability testing, gene ontology and epitope mapping. Using these various criteria, we narrowed it down to prioritize 5 novel drug targets against $S$. flexneri and one vaccine drug targets against all strains of Shigella. Hence, we suggest the identified enzymes as the best putative drug targets for the effective treatment of S. flexneri.
\end{abstract}

Keywords: Essential enzymes, in silico comparative analysis, KEGG database, metabolic pathway analysis, subcellular localization

Received: July 6, 2020 Accepted: December 10, 2020

First published online: December 14, 2020

* Corresponding author Phone: +91-9811099082 E-mail: hinabansal@gmail.com

Supplementary data for this paper are available on-line only at http://jmb.or.kr.

pISSN 1017-7825 elSSN 1738-8872

Copyright(C) 2021 by The Korean Society for Microbiology and Biotechnology

\section{Introduction}

Shigella flexneri is a gram-negative bacterium that belongs to the family Enterobacteriaceae. The genus Shigella can be classified into 4 species: Shigella flexneri, Shigella boydii, Shigella sonnei and Shigella dysenteriae. The species can be further diversified into different serotypes dependent on biochemical contrasts and variety of Oantigen. S. flexneri is separated into 13 serotypes. Shigellosis is an increasing cause of concern in developing nations as it causes more than 170 million estimated deaths each year, with the primary victims being children below 5 years of age [1]. S. flexneri is one of the most lethal organisms afflicting developing countries [2]. The mode of transmission is the fecal-oral route and a number as low as 10-100 can cause severe infections in human. Shigella species attack the epithelium of the colon and rectum in primates and people, causing the intense aggravation of the mucosa which is associated with shigellosis. Without compelling medications, shigellosis patients may develop auxiliary conditions, for example, septicaemia, pneumonia and haemolytic uremic disorder. The increased prevalence in developing countries is due to lack of food and water hygiene. The pathogenesis of $S$. flexneri is rapid in the system, as once it enters the colonial epithelium, it leads to very severe inflammation in the mucosal region. Traditional treatment of the disease condition begins with rehydration and antibiotics. However, there have been numerous reports in the last decade that the strain has become multi-drug resistant [3-6]. Over the last decade, Shigella has acquired plasmid-encoded resistance to the traditional drugs provided to patients as the first line of treatment. Hence, newer approaches and research must be undertaken to combat the increasing drug resistance.

With the advent of the post-genomic era, since the human genome project was completed successfully, there has been a revolution in the development of drug-designing approaches. The experimental approaches for drug designing are time consuming and costly. Host pathogen interactions can be studied by identifying the non- 
homologous proteins for host and pathogens. These proteins can be treated as targets in the drug discovery process. Pathway analysis using comparative methods has been the most sought-after approach in the last decade. A number of studies have been published in the past with reference to different pathogens on the basis of metabolic pathway analysis and protein-protein interaction studies [7-11]. However, an in-depth Gene Ontology analysis and epitope prediction to identify the putative targets have not been performed in previous work. These steps are essential because while Gene Ontology studies help us in identifying important features such as molecular function and cellular processes for a better understanding of the targets, epitope prediction helps us in identifying the main antigenic properties of the micro organisms. Our results indicate better reliability of the predicted targets for further validation by experimental studies. In the current study, we performed a metabolic pathway comparison between the pathogen and the host to identify essential enzymes for the survival of the bacteria and based on our prediction we have identified potential drug targets for the pathogen. The process began with the identification of metabolic pathways from the KEGG database for both the host and the pathogen. Next, we manually compared the pathways to identify those unique to the pathogen. Further, all enzymes for the unique pathways were extracted and submitted to an online tool for identification. The identified essential enzymes were further screened to determine the feasibility of therapeutic targets that were predicted and analyzed using novel drug target identification, cellular localization, gene ontology analysis and epitope prediction.

\section{Methodology}

A schematic representation of the methodology is given in Fig. 1.

\section{Comparative Analysis of Host and Pathogen Metabolic Pathways}

The extraction of the metabolic pathways was done using the KEGG [12] pathway database for the host Homo sapiens and the pathogen S. flexneri. Pathways were then manually compared to identify those that were unique only to the pathogen. The criterion for selecting the pathways was based on whether the pathway was not present in the host. human but was present in the pathogen and unique to $S$. flexneri. The identification numbers of all metabolic pathways from both organisms were extracted from the database. A manual comparison was then conducted by placing the name of each individual pathway of the pathogen against the pathways of the host, H. sapiens. According to the KEGG database annotations, pathways that were absent in humans but did appear in the pathogen were considered unique to S. flexneri. Further, the enzymes that were involved in these unique pathways were extracted from the KEGG database and sequence retrieval was done from the NCBI database in the FASTA format.

\section{Identification of Non-Homologous Essential Genes}

Protein sequences extracted in the FASTA format which were part of the unique pathways were submitted to the Geptop tool [13] to identify their essentiality in the pathogen. Geptop is a server used to identify essential genes of bacterial species by comparing their orthology and phylogeny with the essential gene database DEG. These essential genes were searched against proteins from the human RefSeq protein database for non-homology using NCBIBLASTP [14]. Proteins having identity below $35 \%$ and an E-value cutoff of 0.005 were selected as non-host proteins.

\section{Protein Network Analysis}

Functional interactions take place between genes/proteins and provide fundamental knowledge for cellular processing and systematic characterization, which play a vital role in molecular systems biology [15]. The PPI (Protein-Protein Interaction) network of all non-homologous proteins was built in Cytoscape v3.7.2 [16] using the STRING app. The interaction of network data was analyzed by network analyzer module [17]. The detection of the functional module of non-homologous proteins was done by MCODE plugin [18] under the degree cutoff $=2$, maximum depth $=100, \mathrm{k}$-core $=2$, and node score cutoff $=0.2$. The uppermost hierarchical module was chosen as the utmost possible metabolic functional associations of the interacting proteins selected for further analysis.

\section{Subcellular Localization and Identification of Novel Drug Targets}

Subcellular localization of the essential non-human proteins selected from network analysis was predicted by PSORTb v3.0.2 [19] and CELLO v2.518. Transmembrane proteins were identified by TMHMM Server v. 2.0. TMHMM server is based on the hidden Markov model. To find the most probable topology of a membrane protein, N-best algorithm was used. Proteins with a transmembrane helix predicted as having fewer than 50 amino acid residues from the $\mathrm{N}$ terminus were extracted as likely candidates for signal peptides. In addition, if a cleavage site was predicted with a probability $>0.5$, the predicted signal peptide was cleaved off and the prediction was redone [20,21]. Only cytoplasmic and transmembrane proteins were selected as novel drug targets [23]. Further, the DrugBank databases [22] was used to identify novel targets amongst selected potential targets with an E-value of less than 10-5, sequence identity greater than 35\%, and a bit score greater than 100 .

\section{Gene Ontology and B-Cell Epitope Prediction}

Novel drug targets of Shigella flexneri are ideal as drug candidates. Two-step methodology was performed. to analyze the drug targets identified through the work. As the first step, the DAVID Functional Annotation Tool was used for Gene Ontology analysis [24,25]. The complete list of 26 proteins identified was uploaded for Gene Ontology Analysis under the categories of Cellular Compartment (CC), Biological Process (BP), Molecular Function (MF), and InterPro Terms. Further, the B-cell epitopes of the proteins were predicted by ABCpred Prediction Server [26], threshold $=0.91$, window length $=20$. 


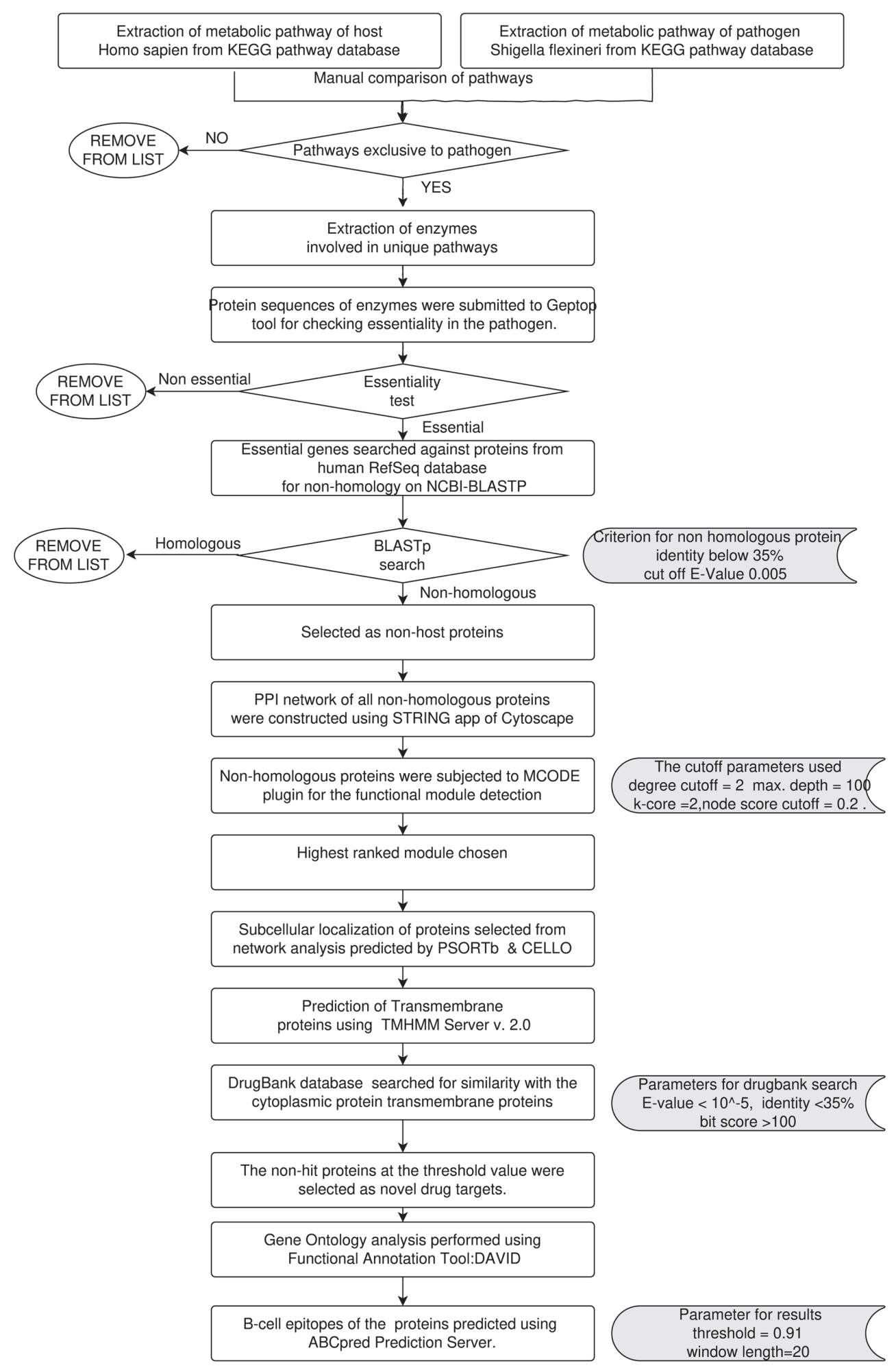

Fig. 1. A schematic representation of the methodology.

\section{Results}

Comparative Metabolic Pathway Analysis

Metabolic pathways of host and pathogen were examined using the KEGG database. Relative investigation was executed manually for the recognizable proof of pathways exclusive to S. flexneri. Metabolic pathways that are available in both the host and the pathogen are distinguished as normal pathways and those which are available as 
Table 1. List of metabolic pathways unique to Shigella flexneri.

\begin{tabular}{|c|c|c|}
\hline S. No. & Pathway ID & Pathway Name \\
\hline 1 & 00660 & C5-Branched dibasic acid metabolism \\
\hline 2 & 00680 & Methane metabolism \\
\hline 3 & 00121 & Secondary bile acid biosynthesis \\
\hline 4 & 00300 & Lysine biosynthesis \\
\hline 5 & 00460 & Cyanoamino acid metabolism \\
\hline 6 & 00473 & D-Alanine metabolism \\
\hline 7 & 00540 & Lipopolysaccharide biosynthesis \\
\hline 8 & 00550 & Peptidoglycan biosynthesis \\
\hline 9 & 00903 & Limonene and pinene degradation \\
\hline 10 & 00281 & Geraniol degradation \\
\hline 11 & 00523 & Polyketide sugar unit biosynthesis \\
\hline 12 & 01053 & Biosynthesis of siderophore group nonribosomal peptides \\
\hline 13 & 00332 & Carbapenem biosynthesis \\
\hline 14 & 00261 & Monobactam biosynthesis \\
\hline 15 & 00521 & Streptomycin biosynthesis \\
\hline 16 & 00525 & Acarbose and validamycin biosynthesis \\
\hline 17 & 00401 & Novobiocin biosynthesis \\
\hline 18 & 00362 & Benzoate degradation \\
\hline 19 & 00627 & Aminobenzoate degradation \\
\hline 20 & 00364 & Fluorobenzoate degradation \\
\hline 21 & 00625 & Chloroalkane and chloroalkene degradation \\
\hline 22 & 00361 & Chlorocyclohexane and chlorobenzene degradation \\
\hline 23 & 00623 & Toluene degradation \\
\hline 24 & 00633 & Nitrotoluene degradation \\
\hline 25 & 00930 & Caprolactam degradation \\
\hline 26 & 00626 & Naphthalene degradation \\
\hline
\end{tabular}

having pathogenicity and not in the host are deemed as novel pathways. A total of 85 metabolic pathways were extracted from the pathogen and among these 26 metabolic pathways (Table 1) were identified as unique to S. flexneri.

\section{Identification of Essential Genes}

All the enzymes associated with these 26 unique pathways were identified and examined for their essentiality to the pathogen by using the tool Geptop 2.0. A total of 4179 genes are submitted and 395 of them are predicted as essential genes. Their accession no. and name were accessed from NCBI. BLASTP search was performed specifically against $H$. sapiens with an E-value threshold of 0.005 and an identity percentage of $\leq 35 \%$ were considered as non-host proteins which do not have human homologues. Out of 395 essential enzymes, 269 were found to be non-host as they did not show homology with human (Suppl. 1).

\section{Protein-Protein Interaction Network Analysis}

Functional associations between 269 non-host, essential genes of S. flexneri were studied using STRING app of Cytoscape v3.7.2 (Fig. 2). Interacting proteins with high confidence scores were visualized using MCODE plugin to predict a protein-protein complex data set. The molecular complex detection (MCODE) is a Cytoscape plugin that enabled detection of clusters in large protein interaction networks. Clusters obtained from the protein interaction network can be considered as protein complexes and functional modules, which can be identified as highly interconnected subgraphs [18]. Finding precisely the important interacting enzymes as network clusters provides insights into the exploration of potential drug targets. The highest ranked module having 57 nodes and 1444 edges was chosen as the utmost possible metabolic functional associations between identified proteins.

\section{Prediction of Subcellular Localization and Identification of Novel Drug Targets}

Subcellular localization of 57 proteins revealed that 50 proteins were cytoplasmic, 4 were extracellular and 3 were transmembrane proteins (Fig. 2). Next, unveiling of novel targets was conducted using the DrugBank database. Proteins showing no matching hits against the DrugBank database at the threshold were nominated as novel drug targets. The results revealed 26 proteins that were uniquely involved in pathogen-specific unique pathways (Table 2).

\section{Gene Ontology and B-Cell Epitope Mapping}

Gene Ontology analysis revealed interesting information on the drug proteins identified. Under the category of Biological Process, it was observed that $73 \%$ of the identified targets belonged to the "Translation" process. Gene Ontology for Molecular Function revealed that $53 \%$ of the proteins were associated with "structural constituent of ribosome". Under the Cellular Component section, most of the drug targets belonged to the "ribosome" compartment. Further, performing an InterPro scan revealed an equal distribution amongst 4 major protein 


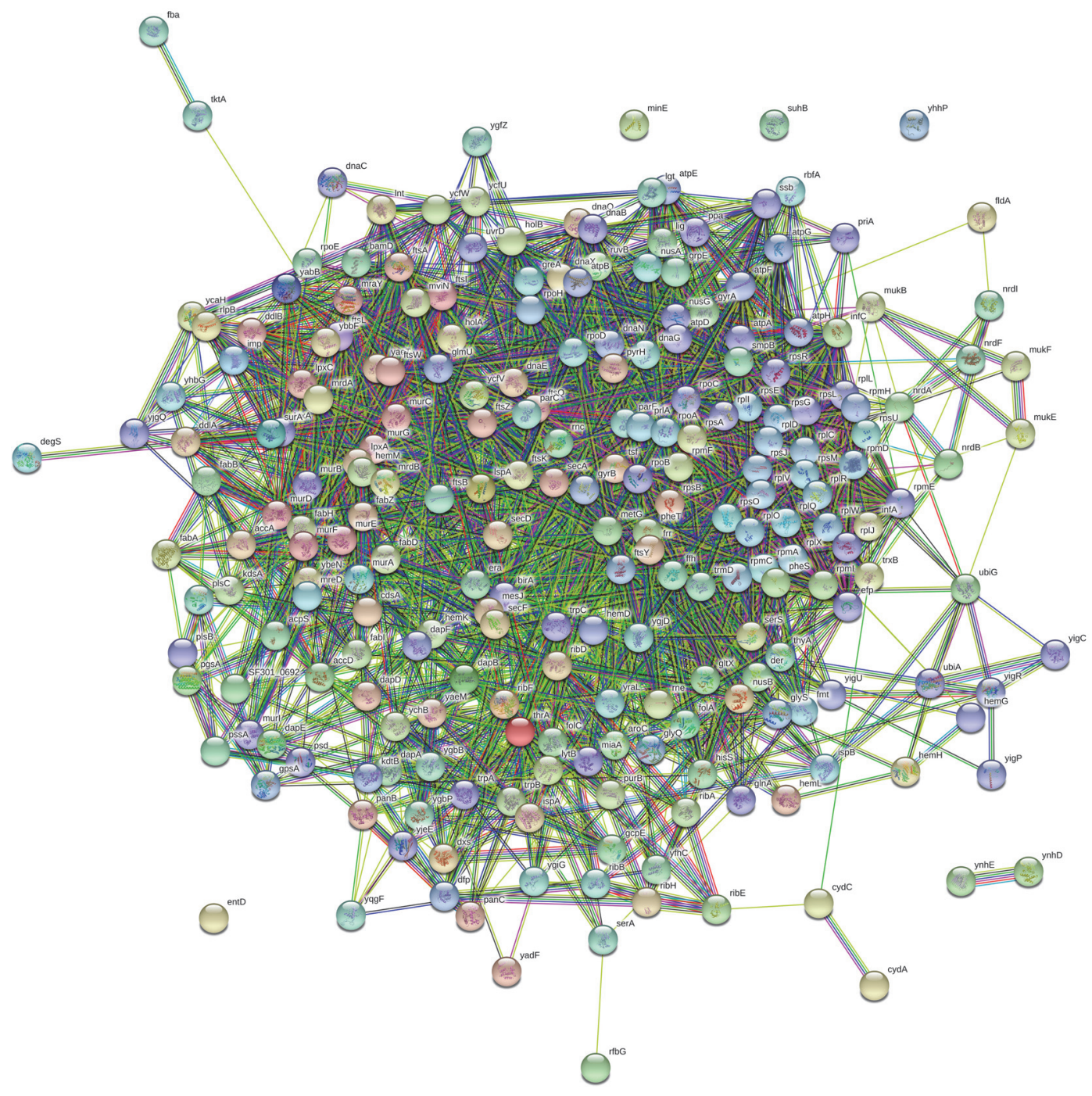

Fig. 2. Protein-protein interaction network of non-host essential proteins from Shigella flexneri.

signatures: Ribosomal protein L2 domain 2, Translation protein SH3 like domain, Nucleic acid-binding domain, KOW and RNA-binding domain S1 (Fig. 4). In addition, B-cell epitope mapping was performed by ABCpred Prediction Server based on artificial neural network. The predicted B-cell epitopes were ordered based on their score attained. The top five highest ranked epitopes with a score of $>9.0$ were selected as highest probable epitope (Table 3).

PROTEIN LOCALIZATION

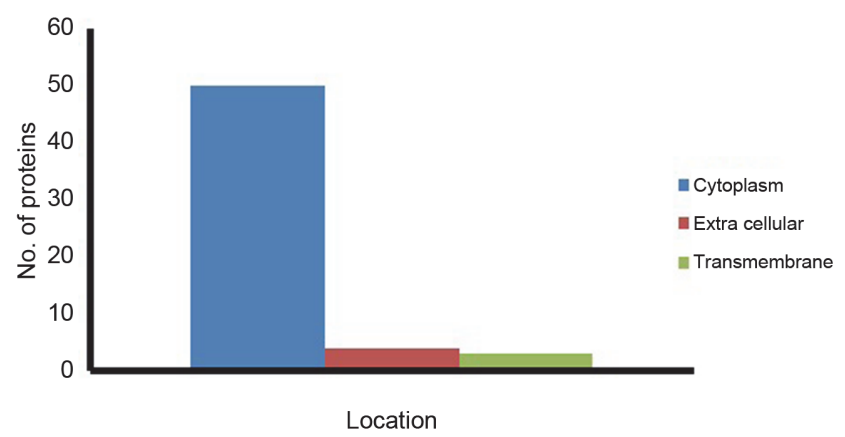

Fig. 3. Prediction of subcellular localization. 
Table 2. List of proteins selected as novel drug targets.

\begin{tabular}{|c|c|c|c|c|}
\hline S No. & Accession No. & Protein names & Subcellular localization & Novel drug targets \\
\hline 1 & NP_710014 & Elongation factor P (EF-P) & Cytoplasm & No Hits \\
\hline 2 & NP_706048 & Cell division protein FtsQ & Transmembrane & No Hits \\
\hline 3 & NP_706769 & Translation initiation factor IF-1 & Cytoplasm & No Hits \\
\hline 4 & NP_707396 & Translation initiation factor IF-3 & Cytoplasm & No Hits \\
\hline 5 & NP_709777 & Transcription termination & Cytoplasm & No Hits \\
\hline 6 & NP_709088 & Protein translocase subunit $\mathrm{SecY}$ & Transmembrane & No Hits \\
\hline 7 & NP_710066 & 50S ribosomal protein L9 & Cytoplasm & No Hits \\
\hline 8 & NP_709089 & 50S ribosomal protein L15 & Cytoplasm & No Hits \\
\hline 9 & NP_709082 & 50 S ribosomal protein L17 & Cytoplasm & No Hits \\
\hline 10 & NP_709092 & 50 S ribosomal protein L18 & Cytoplasm & No Hits \\
\hline 11 & NP_708985 & 50 S ribosomal protein $\mathrm{L} 21$ & Cytoplasm & No Hits \\
\hline 12 & NP_709106 & 50 S ribosomal protein L23 & Cytoplasm & No Hits \\
\hline 13 & NP_709097 & 50 S ribosomal protein L24 & Cytoplasm & No Hits \\
\hline 14 & NP_709416 & 50 S ribosomal protein $\mathrm{L} 28$ & Cytoplasm & No Hits \\
\hline 15 & NP_709100 & 50 S ribosomal protein L29 & Cytoplasm & No Hits \\
\hline 16 & NP_709090 & 50 S ribosomal protein L30 & Cytoplasm & No Hits \\
\hline 17 & NP_709740 & 50 S ribosomal protein $\mathrm{L} 31$ & Cytoplasm & No Hits \\
\hline 18 & NP_707005 & 50 S ribosomal protein $\mathrm{L} 32$ & Cytoplasm & No Hits \\
\hline 19 & NP_709497 & 50 S ribosomal protein L34 & Cytoplasm & No Hits \\
\hline 20 & NP_707397 & 50 S ribosomal protein L35 & Cytoplasm & No Hits \\
\hline 21 & NP_709083 & DNA-directed RNA polymerase subunit alpha & Cytoplasm & No Hits \\
\hline 22 & NP_706830 & $30 S$ ribosomal protein $\mathrm{S} 1$ & Cytoplasm & No Hits \\
\hline 23 & NP_708875 & 30 S ribosomal protein $\mathrm{S} 21$ & Cytoplasm & No Hits \\
\hline 24 & NP_709776 & Protein translocase subunit $\mathrm{SecE}$ & Transmembrane & No Hits \\
\hline 25 & NP_706115 & Elongation factor Ts (EF-Ts) & Cytoplasm & No Hits \\
\hline 26 & NP_708456 & Ribosome maturation factor RimM & Cytoplasm & No Hits \\
\hline
\end{tabular}

\section{GO: BIOLOGICAL PROCESS}

- translation

mprotein transport by the Sec complex

- intracellulas protein transmembrane transport

a protein target:ing

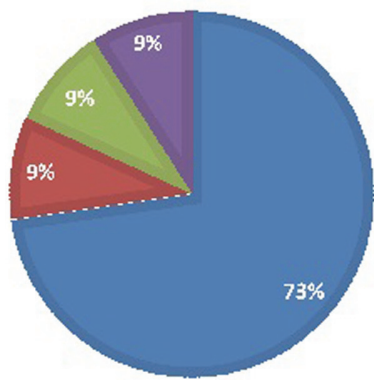

GO:CELLULAR

COMPARTMENT

- ribosome arge ribosomal subunit

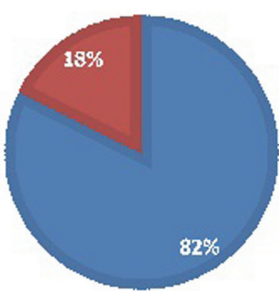

\section{GO: MOLECULAR}

FUNCTION

- structural constituent of riboscme

口rRNA biading

ribosome binding

E translation initiation factor activity

- translation elo:ngation factor activity

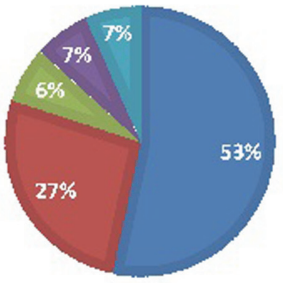

\section{INTERPRO TERMS}

Ribcsoral protein L2 dome in 2

- Translation protein S113-like domain

nucleic acid-binding, 0:3-fold

-KOW

RNA-binding domain, 51

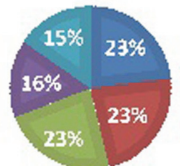

Fig. 4. Gene Ontology analysis using Functional Annotation Tool: DAVID. 
Table 3. List of predicted epitopes having score value greater than threshold.

\begin{tabular}{ccllcc}
\hline Rank & Accession No. & \multicolumn{1}{c}{ Protein name } & \multicolumn{1}{c}{ Sequence } & Start position & Score \\
\hline 1 & NP_710014 & Elongation factor P (EF-P) & KVPLFVQIGEVIKVDTRSGE & 199 & 0.96 \\
2 & NP_709083 & $\begin{array}{l}\text { DNA-directed RNA } \\
\text { polymerase subunit alpha }\end{array}$ & VILTLNKSGIGPVTAADITH & 3643 & 0.94 \\
3 & NP_706830 & 30S ribosomal protein S1 & VTGVINGKVKGGFTVELNGI & 4018 & 0.93 \\
4 & NP_706048 & Cell division protein FtsQ & AAMTARRSWQLTLNNDIKLN & 458 & 0.92 \\
4 & NP_709106 & 50S ribosomal protein L23 & STAMEKSNTIVLKVAKDATK & 2582 & 0.92 \\
5 & NP_706115 & Elongation factor Ts (EF-Ts) & NMRKSGAIKAAKKAGNVAAD & 4827 & 0.91 \\
\hline
\end{tabular}

\section{Discussion}

The present study focused on subtractive genome analysis (Fig. 5) that led to identify the proteins which can be used as potential targets for drug development against the pathogenicity of $S$. flexneri. The rationale of picking targets using computational analysis depends on deciphering those proteins that are unique to the pathogen. Designing a drug exclusively for such a target will affect only the pathogen but won't interfere with any aspects of the host biology. Therefore, using extensive in silico analysis, we identified broad-spectrum antibiotic targets and proposed elongation factor P, DNA-directed RNA polymerase subunit alpha, 30S ribosomal protein S1, 50S ribosomal protein L23, elongation factor Ts, cell division protein FtsQ as novel drug targets. The analysis revealed that protein elongation factor P, DNA-directed RNA polymerase subunit alpha, 30 S ribosomal protein S1, cell division protein FtsQ, 50S ribosomal protein L23 and elongation factor Ts are cytoplasmic and can be used as potential drug targets against $S$. flexneri. However, protein cell division protein FtsQ is a membrane protein and can work as a suitable vaccine drug target against all strains of Shigella. Further, it has been observed that all these proteins have been involved significantly in the major metabolic pathways unique to the pathogen like lysine biosynthesis, lipopolysaccharide biosynthesis and peptidoglycan biosynthesis. Moreover, 30S ribosomal protein $\mathrm{S} 1$ is vital for binding with the mRNA in Shigella; thus, enabling recognition of the initiation point. The target protein is needed to translate mRNA with a short Shine-Dalgarno (SD) purine-rich sequence. Part of the 30S ribosomal subunit such as some nascent polypeptide chains can cross-link to this protein. Also, 50S ribosomal protein L23 is the underlying gathering protein and ties with the $23 \mathrm{~S}$ rRNA. This protein target frames the center docking site as a requisite to bind with ribosome. One of the early assembly proteins, it binds $23 \mathrm{~S}$ rRNA. It is an important target since it is one of the proteins that surrounds the polypeptide exit tunnel on the outside of the ribosome and forms the main docking site for trigger factor binding to the ribosome. It is an important contact for protein L29, and trigger factor when it is bound to the ribosome. The main function of DNA-directed RNA polymerase subunit alpha is to catalyze translation of DNA into RNA utilizing four ribonucleoside triphosphates as substrates. DNA-dependent RNA polymerase catalyzes the transcription of DNA into RNA using the four ribonucleoside triphosphates as substrates and is a homodimer. The RNAP catalytic core consists of 2 alpha, 1 beta, 1 beta' and 1 omega subunit. When a sigma factor is associated with the core the holoenzyme is formed, which can initiate transcription. The N-terminal domain is essential for RNAP assembly and basal transcription, whereas the C-terminal domain is involved in interaction with transcriptional regulators and with upstream promoter elements. Elongation factor $\mathrm{P}$ is mainly engaged with the synthesis of peptide bonds and reduces ribosome, slowing down protein which requires change of Lys-34. Elongation factor Ts is linked with the EF-Tu. GDP complex prompts the trading of GDP to GTP. It stays bound to the aminoacyl-tRNA.EF-Tu.GTP complex up to the GTP hydrolysis stage on the ribosome. Elongation factors are proteins that perform significant roles during the elongation cycle of protein biosynthesis on the ribosome. They alleviate ribosome pausing at polyproline (PPX) motifs by enabling peptide bond formation. In the absence of these proteins, PPX peptide bond formation

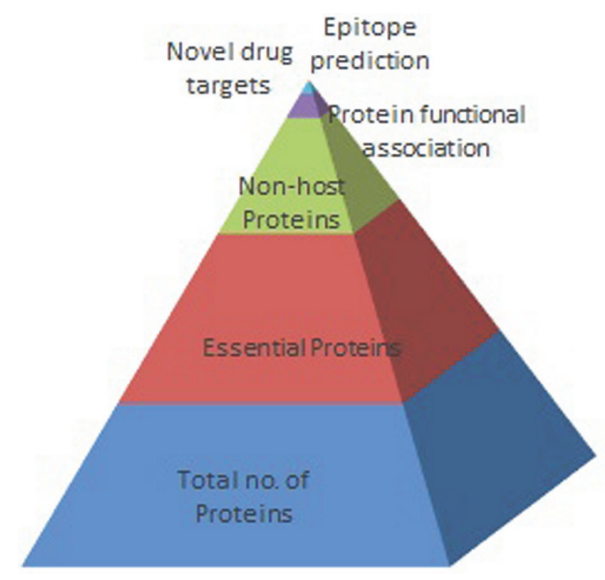

Fig. 5. Subtractive dataset in Shigella flexneri. 
can limit translation rate, leading to pleotropic phenotypes with slowed growth, increased antibiotic sensitivity, and loss of virulence. Hence, these proteins are the key targets of several antibiotics. Cell division protein FtsQ is a fundamental cell division protein. It performs a key role in the assembly of the divisome, a large protein complex that regulates bacterial cell division. It formulates a stable trimeric complex with FtsB and FtsL, which is essential for successful cell division $[27,28]$. The interactions with FtsB and FtsL mostly take place in the periplasmic domains of these proteins, indicating that drugs targeting these interactions would only have to cross the outer membrane of the bacterium. Hence it can be suggested as a potential vaccine candidate for antibacterial treatment $[29,30]$.

\section{Conclusions}

Shigella flexneri is a gram-negative bacterium that is a member of the family Enterobacteriaceae. It is the most endemic species and the most lethal for developing countries. Traditional treatment of the disease condition begins with rehydration and antibiotics. However, there have been numerous reports in the last decade suggesting that the strain has become multi-drug resistant. Over the last decade, Shigella has acquired plasmid-encoded resistance to the traditional drugs that were provided to patients as the first line of treatment. Here, we employed comparative metabolic pathway analysis for the identification of essential enzymes for the survival of the bacteria and based on our prediction we are able to propose potential drug targets for the pathogen. In conclusion, we can suggest five essential enzymes, elongation factor P, DNA-directed RNA polymerase subunit alpha, 30S ribosomal protein S1, 50S ribosomal protein L23 and elongation factor Ts as the best putative drug targets against the pathogenesis of Shigella flexneri and cell division protein FtsQ as the vaccine drug targets against all strains of Shigella. To the best of our understanding, the research outcome from our work could highlight a novel approach and identify drug targets to overcome the infection caused by the pathogen. In the future, whole genome proteome sequence data can be retrieved for all the strains of Shigella and further core proteomic analysis can be performed for the dataset, leading to improved and more enhanced applications of this work.

\section{Acknowledgments}

The authors are highly grateful to founder President Dr. Ashok K Chauhan and Chancellor Mr. Atul Chauhan Amity University Uttar Pradesh, Noida, India for providing necessary support and facilities.

\section{Conflict of Interest}

The authors have no financial conflicts of interest to declare.

\section{References}

1. Lanata CF, Fischer-Walker CL, Olascoaga AC, Torres CX, Aryee RB. 2013.Global causes of diarrheal disease mortality in children $<5$ years of age: a systematic review. PLoS One 8: e72788.

2. Mandal J, VG, Emelda, Subhash MS, Parija SC. 2012. The recent trends of Shigellosis: a JIPMER perspective. J. Clin. Diagn. Res. 6: $1474-1477$.

3. von Seidlein L, Kim DR, Ali M, Lee H, Wang X, Thiem VD, et al. 2006. A multicentre study of Shigella diarrhoea in six Asian countries: disease burden, clinical manifestations, and microbiology. PLoS Med. 3: e353.

4. Naheed A, Kalluri P, Talukder KA, Faruque ASG, Khatun F, Nair GB, et al. 2004. Fluoroquinolone-resistant Shigella dysenteriae type 1 in northeastern Bangladesh. Lancet Infect. Dis. 4: 607-608.

5. Sivapalasingam S, Nelson JM, Joyce K, Hoekstra M, Angulo FJ, Mintz ED. 2006. High prevalence of antimicrobial resistance among Shigella isolates in the United States tested by the National Antimicrobial Resistance Monitoring System from 1999 to 2002. Antimicrob. Agents Chemother. 50: 49-54

6. Dutta D, Bhattacharya MK, Dutta S, Datta A, Sarkar D, Bhandari B, et al. 2003. Emergence of multidrug-resistant Shigella dysenteriae type 1 causing sporadic outbreak in and around Kolkata, India. J. Health Popul. Nutr. 21: 79-80.

7. Uddin R, Sufian M. 2016. Core proteomic analysis of unique metabolic pathways of Salmonella enterica for the identification of potential drug targets. PLoS One 11: e0146796.

8. Hema K, Priyadarshini VI, Pradhan D, Munikumar M, Sandeep S, Pradeep N, et al. 2015. Identification of putative drug targets and vaccine candidates for pathogens causing atherosclerosis. Biochem. Anal. Biochem. 4: 1

9. Vetrivel U, Subramanian G, Dorairaj S. 2011. A novel in silico approach to identify potential therapeutic targets in human bacterial pathogens. Hugo J. 5: 25-34.

10. Hasan MA, Khan MA, Sharmin T, Hasan Mazumder MH, Chowdhury AS. 2016. Identification of putative drug targets in Vancomycin-resistant Staphylococcus aureus (VRSA) using computer aided protein data analysis. Gene 575: 132-143.

11. Munikumar M, Priyadarshini IV, Pradhan D, Sandeep S, Umamaheswari A, and Vengamma B. 2012. In silico identification of common putative drug targets among the pathogens of bacterial meningitis. Biochem. Anal. Biochem. 1: 123.

12. Kanehisa M, Goto S, Kawashima S, Nakaya A. 2002. The KEGG databases at GenomeNet. Nucleic Acids Res. 30: $42-46$

13. Wei W, Ning LW, Ye YN, Guo FB. 2013. Geptop: a gene essentiality prediction tool for sequenced bacterial genomes based on orthology and phylogeny. PLoS One 8: e72343.

14. Altschul SF, Gish W, Miller W, Myers EW, Lipman DJ. 1990. Basic local alignment search tool. J. Mol. Biol. 215: 403-410.

15. Szklarczyk D, Franceschini A, Wyder S, Forslund K, Heller D, Huerta-Cepas, J. et al. 2015. STRING v10: protein-protein interaction networks, integrated over the tree of life. Nucleic Acids Res. 43(Database issue): D447-D452.

16. Cline MS, Smoot M, Cerami E, Kuchinsky A, Landys N, Workman C, et al. 2007. Integration of biological networks and gene expression data using Cytoscape. Nat. Protoc. 2: 2366-2382.

17. Assenov Y, Ramírez F, Schelhorn SE, Lengauer T, Albrecht M. 2008. Computing topological parameters of biological networks. Bioinformatics 24: 282-284.

18. Bader GD, Hogue CW. 2003. An automated method for finding molecular complexes in large protein interaction networks. BMC Bioinformatics 4: 2 .

19. Yu NY, Wagner JR, Laird MR, Melli G, Rey S, Lo R, et al. 2010. PSORTb 3.0: improved protein subcellular localization prediction with refined localization subcategories and predictive capabilities for all prokaryotes. Bioinformatics 26: 1608-1615. 
20. Krogh A, Larsson B, von Heijne G, Sonnhammer EL. 2001.Predicting transmembrane protein topology with a hidden Markov model: application to complete genomes. J. Mol. Biol. 305: 567-580.

21. Sonnhammer ELL, von Heijne G, Krogh A. 1998. A hidden Markov model for predicting transmembrane helices in protein sequences. pp. 175-182. In Glasgow J, Littlejohn T, Major F, Lathrop R, Sankoff D, Sensen C, editors, Proceedings of the Sixth International Conference on Intelligent Systems for Molecular Biology, Menlo Park, CA, USA.

22. Wishart DS, Feunang YD, Guo AC, Lo EJ, Marcu A, Grane JR, et al. 2018. DrugBank 5.0: a major update to the DrugBank database for 2018. Nucleic Acids Res. 46(D1): D1074-D1082.

23. Yang H, Qin C, Li YH, Tao L, Zhou J, Vu F, et al. 2016. Therapeutic target database update 2016: enriched resource for bench to clinical drug target and targeted pathway information. Nucleic Acids Res. 44(D1): D1069-D1074.

24. Huang da W, Sherman BT, Lempicki RA. 2009. Systematic and integrative analysis of large gene lists using DAVID bioinformatics resources. Nat. Protoc. $4: 44-57$.

25. Huang da W, Sherman BT, Lempicki RA. 2009. Bioinformatics enrichment tools: paths toward the comprehensive functional analysis of large gene lists. Nucleic Acids Res. 37: 1-13

26. Saha S, Raghava GP.2007. Prediction methods for B-cell epitopes. Methods Mol. Biol. 409: 387-394.

27. Glas M, McLaughlin SH, Roseboom W, Liu F, Koningstein GM, Fish A, et al. 2015. The soluble periplasmic domains of Escherichia coli cell division proteins FtsQ/FtsB/FtsL form a trimeric complex with submicromolar affinity. J. Biol. Chem. 290: 21498-21509.

28. Buddelmeijer N, Beckwith J. 2004. A complex of the Escherichia coli cell division proteins FtsL, FtsB and FtsQ forms independently of its localization to the septal region. Mol. Microbiol. 52: 1315-1327.

29. Den Blaauwen T, Andreu JM, Monasterio O. 2014. Bacterial cell division proteins as antibiotic targets. Bioor. Chem. 55: 27-38.

30. Lock RL, Harry EJ. 2008. Cell-division inhibitors: new insights for future antibiotics. Nat. Rev. Drug Discov. 7: 324-338 